\title{
Interaction of Electromagnetic Wave and Metamaterial with Inductive Type Chiral Inclusions
}

\author{
A. N. Volobuev \\ Samara State Medical University. Department of Medical and Biological Physics \\ Email: volobuev47@yandex.ru
}

\begin{abstract}
The principle of calculation of a plate from a metamaterial with inductive type chiral inclusions is submitted. It is shown that distribution of an electromagnetic wave in such substance can be investigated with the help of using of a chiral parameter and on the basis of a detailed method of calculation. By comparison of two methods the dependence of chiral parameter from frequency of electromagnetic radiation falling on a plate is found. With the help of a detailed method the nonlinear differential equation for potential on the chiral plate is found. It is shown that this equation has solutions as traveling solitary waves and standing waves but not traveling sine waves. The analysis of the received solutions of the nonlinear equation is carried out. Transition from the multiwave solution to the solution as standing waves is graphically shown at reduction of distance between the chiral elements.
\end{abstract}

Keywords: metamaterial, chiral parameter, inductive inclusions, multiwave solution, standing waves.

\section{$1 \quad$ Introduction}

Now the materials (Greek. "meta" outside), i.e. composite materials with the various inclusions distributed both chaotically, and periodically are widely applied in particular in a radio engineering, at designing of space devices, in medicine, etc., $[1,2,3,4]$. Due to these inclusions the received materials have many useful physical, electric, optical and other properties which are not present at natural substances. Among metamaterials are allocated the substances with chiral properties [5] which are capable to rotate a plane of polarization of electromagnetic waves. In optics as analogue of similar substances are the optical active substances, for example, quartz, a glucose solution etc.

However methods of calculation of metamaterials are enough limited [6]. Basically all calculations are based on the solving of the Maxwell's equations and the material equations selected according to a problem.

The existing method has restrictions since average characteristics of metamaterials are usually used only, for example, a chiral parameter.

In the present work attempt of more detailed approach to properties of chiral inclusions into metamaterials is made; the analysis of influence of these properties on interaction of chiral elements with the electromagnetic wave falling on a plate from a metamaterial is carried out.

\section{Standard Method of Calculation of a Metamaterial with an Electromagnetic Wave Interaction}

At research of metamaterials with chiral inclusions on the basis of the Maxwell's equations usually use the material equations including so-called chiral parameter $\chi$. In $[5,6,7]$ the material equations in the following kind are offered:

$$
\begin{aligned}
& \mathbf{D}=\varepsilon_{a} \mathbf{E} \mp i \frac{\chi}{V} \mathbf{H}, \\
& \mathbf{B}=\mu_{a} \mathbf{H} \pm i \frac{\chi}{V} \mathbf{E},
\end{aligned}
$$

where $\boldsymbol{D}$ and $\boldsymbol{B}$ there are an induction of electric and magnetic fields in the electromagnetic wave propagating in a chiral medium, $\boldsymbol{E}$ and $\boldsymbol{H}$ - strength of the electric and magnetic components in wave, 
$\varepsilon_{a}$ and $\mu_{a}$ - absolute electric and magnetic permeability of a chiral medium, $V$ - velocity of an electromagnetic wave in a chiral medium, $\chi$ - a chiral parameter, in this case dimensionless size.

In [7] it is shown that the material equations (1) and (2) can be written down in more simple kind:

$$
\begin{aligned}
& \mathbf{D}=(1 \pm \chi) \varepsilon_{a} \mathbf{E}, \\
& \mathbf{B}=(1 \pm \chi) \mu_{a} \mathbf{H} .
\end{aligned}
$$

In formulas (1) - (4) top signs define the right-turning chiral element, bottom signs - left-turning.

Using (3) and (4) it is possible to show [7] that if a chiral medium has only reactive resistance, the electromagnetic wave in it submits to the wave equations:

$$
\begin{aligned}
& \Delta \mathbf{D}=\left(\frac{1 \pm \chi}{V}\right)^{2} \frac{\partial^{2} \mathbf{D}}{\partial t^{2}}, \\
& \Delta \mathbf{B}=\left(\frac{1 \pm \chi}{V}\right)^{2} \frac{\partial^{2} \mathbf{B}}{\partial t^{2}},
\end{aligned}
$$

where $t$ there is time.

Further us the equation (5) will interest only. Substituting (3) in (5) and transit to scalar potential $\varphi \quad[8]$ we shall find:

$$
\Delta \varphi=\left(\frac{1 \pm \chi}{V}\right)^{2} \frac{\partial^{2} \varphi}{\partial t^{2}} .
$$

Let's search the solution of the equation $(7)$ as:

$$
\varphi-\varphi_{0}=\varphi(\mathbf{r}) \exp (i \omega t)
$$

where $\varphi_{0}$ there is an origin of potential, $\boldsymbol{r}$ - set of the spatial coordinates, $\omega$ - cyclic frequency of an electromagnetic wave falling on substance.

Substituting (8) in (7) we have:

$$
\Delta \varphi(\mathbf{r})+(1 \pm \chi)^{2} k^{2} \varphi(\mathbf{r})=0
$$

where $k=\frac{\omega}{V}$ there is a module of an electromagnetic wave vector.

Solving the equation (9) with use of initial and boundary conditions it is possible to investigate processes of reflection, refraction, diffraction of an electromagnetic wave in a metamaterial.

\section{Detailed Method of Calculation of Metamaterial with Electromagnetic Wave Interaction}

Let's consider a plate of the metamaterial with chiral inclusions of the inductive type. The plate consist of the dielectric in which are included the current-carrying chiral elements as spirals which axis is directed across a plate. The chiral elements are distributed periodically

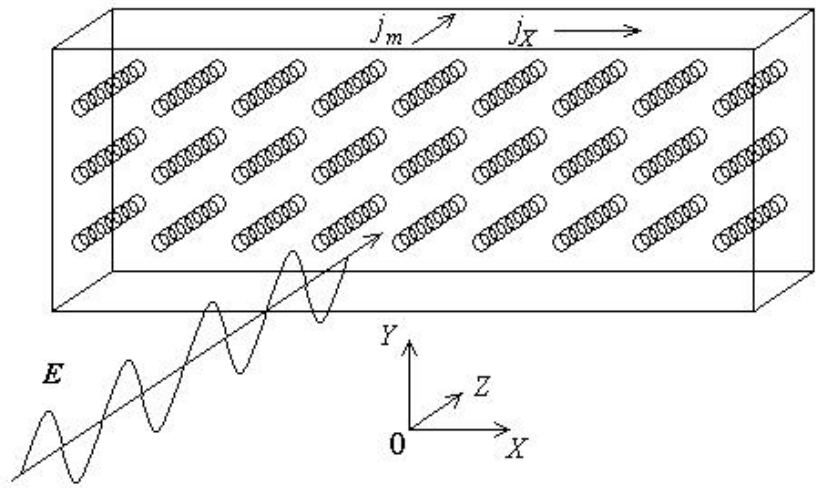

Figure 1. The plate of metamaterials irradiated by an electromagnetic wave 
On fig. 1 the irradiation of a plate by an electromagnetic wave is shown. We assumed that chiral inclusions have no active resistance. The chiral element completely penetrates a plate.

Feature of a plate is the capacity distributed on its surfaces at dot inductive inclusions. Therefore to examine the interaction of separate chiral element having inductance and capacity with an electromagnetic wave is incorrectly.

At the irradiation on the plate there is a potential difference submitting to the equation (7). The density of a current through plate will look like:

$$
j_{m}=C_{m} \frac{\partial \varphi}{\partial t}+\left(\varphi-\varphi_{0}\right) g_{m}
$$

where $C_{m}$ there is capacity of the plate area unit, $\varphi$ - potential on a plate relative to entry level $\varphi_{0}$, $g_{m}$ - electrical conductivity of units of the plate area due to an inductive component.

The first term (10) reflects a capacitor bias current, the second term - an inductive current through the chiral elements.

For spiral chiral element it is possible to write down the equation of a voltage balance:

$$
-L_{i} S_{i} \frac{\partial j_{i}}{\partial t}=\left(\varphi-\varphi_{0}\right)
$$

where $j_{i}$ there is density of a current through the $i$-th chiral element, $L_{i}$ - inductance $i$-th chiral element, $S_{i}$ - the area of plate, falling one chiral element having inductive electrical conductivity $g_{i}$.

The density of a current $j_{i}$ through the chiral element depends on a potential difference on a plate and electrical conductivity it chiral element $g_{i}$ under the formula of the Ohm's law:

$$
j_{i} S_{i}=g_{i}\left(\varphi-\varphi_{0}\right) \text {. }
$$

Substituting (12) in (11) we shall find:

$$
g_{i}=-\frac{\left(\varphi-\varphi_{0}\right)}{L_{i} \frac{\partial \varphi}{\partial t}} .
$$

Electrical conductivity falling unit area of a plate it is equal:

$$
g_{m}=-\frac{\left(\varphi-\varphi_{0}\right)}{S_{i} L_{i} \frac{\partial \varphi}{\partial t}},
$$

where it is taken into account $g_{i}=g_{m} S_{i}$.

Having substituted (14) in (10) we shall find:

$$
j_{m}=C_{m} \frac{\partial \varphi}{\partial t}-\frac{\left(\varphi-\varphi_{0}\right)^{2}}{S_{i} L_{i} \frac{\partial \varphi}{\partial t}}
$$

Using $C_{i}=C_{m} S_{i}$ - the capacity of the plate falling one chiral element, and designating $\omega_{0}^{2}=\frac{1}{C_{i} L_{i}}-$ square of the chiral element natural frequency, we shall find:

$$
\frac{j_{m}}{C_{m}} \frac{\partial \varphi}{\partial t}=\left(\frac{\partial \varphi}{\partial t}\right)^{2}-\left(\varphi-\varphi_{0}\right)^{2} \omega_{0}^{2} .
$$

Let's consider a plate consisting of chiral elements one lines, fig. 2. 


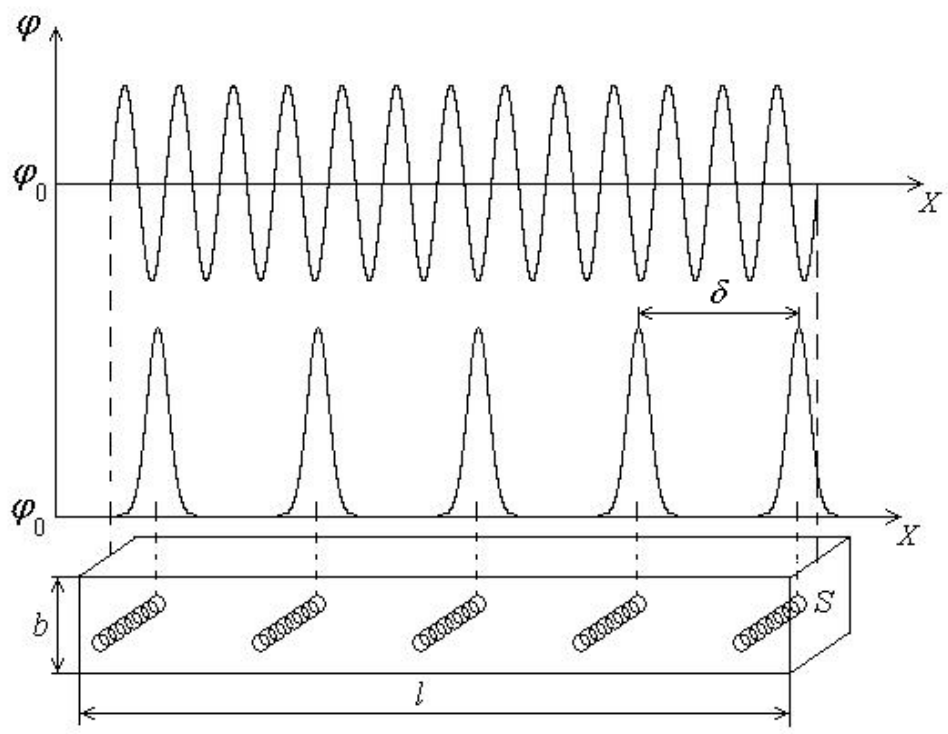

Figure 2. The single-row chiral plate

Along this plate the inductive current flows.

The law of an electromagnetic induction for this current looks like:

$$
-L \frac{\partial I_{X}}{\partial t}=\varphi-\varphi_{0}
$$

where $I_{X}=\gamma_{X} \frac{S}{l}\left(\varphi-\varphi_{0}\right)$ there is a longitudinal inductive current, $\gamma_{X}$ - specific inductive electrical conductivity of a single-row plate, $L$ - its inductance, $S$ - the area of cross-section of a single-row plate, $l$ - its length.

Hence:

$$
-\gamma_{X} S L_{1} \frac{\partial \varphi}{\partial t}=\varphi-\varphi_{0}
$$

where $L_{1}=\frac{L}{l}$ there is inductance of a single-row plate unit of length.

Under the Ohm's law for density of a longitudinal current we have:

$$
j_{X}=-\gamma_{X} \frac{\partial \varphi}{\partial X} .
$$

Hence:

$$
d j_{X}=-\gamma_{X} \frac{\partial^{2} \varphi}{\partial X^{2}} d X
$$

Having divided (20) on (18), and having reduced on $\gamma_{X}$ we shall find:

$$
d j_{X}=\frac{\varphi-\varphi_{0}}{S L_{1} \frac{\partial \varphi}{\partial t}} \frac{\partial^{2} \varphi}{\partial X^{2}} d X .
$$

On the other hand taking into account that a longitudinal current is defined only presence of a crosssection current (or on the contrary) we have:

$$
S d j_{X}=j_{m} b d X
$$

where $b$ there is width of a single-row plate.

Substituting (21) in (22) we have: 


$$
j_{m}=\frac{\varphi-\varphi_{0}}{L_{1} b \frac{\partial \varphi}{\partial t}} \frac{\partial^{2} \varphi}{\partial X^{2}} .
$$

Further substituting (23) in (16) we shall find:

$$
\frac{\varphi-\varphi_{0}}{C_{m} L_{1} b} \frac{\partial^{2} \varphi}{\partial X^{2}}=\left(\frac{\partial \varphi}{\partial t}\right)^{2}-\left(\varphi-\varphi_{0}\right)^{2} \omega_{0}^{2} .
$$

Taking into account $C_{1}=C_{m} b$ - capacity of a single-row plate unit of length and $V^{2}=\frac{1}{C_{1} L_{1}}$ - a square of velocity along a plate of an electromagnetic field we have:

$$
V^{2}\left(\varphi-\varphi_{0}\right) \frac{\partial^{2} \varphi}{\partial X^{2}}=\left(\frac{\partial \varphi}{\partial t}\right)^{2}-\left(\varphi-\varphi_{0}\right)^{2} \omega_{0}^{2}
$$

The nonlinear equation (25) for chiral medium was first obtained in [9].

The nonlinear equation (25) can be transformed to a kind correct for spatial geometry:

$$
V^{2} \Delta \varphi+\omega_{0}^{2}\left(\varphi-\varphi_{0}\right)=\frac{1}{\varphi-\varphi_{0}}\left(\frac{\partial \varphi}{\partial t}\right)^{2}
$$

Linearization of the equations (26) can be carried out by a ratio (8):

$$
\Delta \varphi(\mathbf{r})+k_{S}^{2} \varphi(\mathbf{r})=0,
$$

where is $k_{S}^{2}=\frac{\omega_{0}^{2}+\omega^{2}}{V^{2}}=k_{0}^{2}+k^{2}$, where $k_{S}$ there is a wave number of an electromagnetic wave in chiral medium.

Let's notice that the nonlinear equation similar (25) and (26) arises at research of a self-induced transparency in substance [10], and of the nervous impulse propagation (sequence of action potentials) on a nervous fiber $[11,12]$.

\section{Various Kinds of the Equation Solution of a Metamaterial and an Electromagnetic Wave Interaction}

The equations (27) and (9) reflect the same physical process - propagation of electromagnetic oscillations on the chiral plate. Distinction consists that at a deduction (27) as against (9) was not necessity to use the material equations (1) - (4) i.e. the chiral parameter was not used.

On the basis of the equations (27) and (9) identity it is possible to put down:

$$
k_{S}^{2}=k_{0}^{2}+k^{2}=(1+\chi)^{2} k^{2} .
$$

Further for definiteness are assumed the right-rotating chiral elements.

Hence the chiral parameter can be written down as:

$$
\chi=\sqrt{1+\frac{k_{0}^{2}}{k^{2}}}-1
$$

If $k_{0} \ll k$ or $\omega_{0} \ll \omega$ (natural frequency of chiral medium is much less than frequency of a falling electromagnetic wave) the formula (29) becomes simpler:

$$
\chi=\frac{k_{0}^{2}}{2 k^{2}}=\frac{\omega_{0}^{2}}{2 \omega^{2}} .
$$

Let's notice that quantum calculation of an optical active substance $[13,14]$ results to the formula for chiral parameter:

$$
\chi=\frac{2 V \eta}{3 \hbar} \frac{\omega_{0 j}}{\omega_{0 j}^{2}-\omega^{2}}
$$


where $\hbar$ there is Planck's reduced constant, $\eta$ - size proportional to product of the real parts electric and magnetic dipole moments of an optical active molecule power transition excited by a light of the wave given length, $\omega_{0 j}$ - in this case the frequency corresponding to power transition $0 \rightarrow j$ [15].

The increase in a degree of frequency dependence $\omega_{0}$ up to square-law in the formula (30) in comparison with (31) is characteristic at transition from quantum area in classical.

On fig. 2 the illustrative graph of the potential oscillations on the chiral plate is shown according to the oscillatory solutions satisfying the equations (9) and (27). Character of oscillations will be investigated below.

\subsection{The Solution as Solitary Waves}

The nonlinear equation (25) has solution as a solitary traveling wave:

$$
\varphi-\varphi_{0}=\varphi_{\max } \exp \left(-\frac{\left(k_{0}\left(X-X_{0}\right) \pm \omega_{0}\left(t-t_{0}\right)\right)^{2}}{2}\right)
$$

where $k_{0}=\frac{\omega_{0}}{V}$ there is a wave number of a natural traveling wave in the chiral medium, $\varphi_{\max }-$ a peak value of potential $\varphi-\varphi_{0}, X_{0}$ - coordinate of the chiral element center, and accordingly a maximum (center) of a wave impulse, $t_{0}$ - a time of achievement of this maximum. The sign a minus concerns to a wave spreading from left to right, and sign plus from right to left.

Growth of potential above chiral inclusions, fig. 2, is caused by proportionality of the chiral inclusions reactance their inductivities $\varphi-\varphi_{0} \sim X_{L i}=\omega L_{i}$.

From the analysis of both curves it is possible to conclude that the top curve, fig. 2, concern to often enough inclusions of the chiral elements in a plate, and bottom to more rare. Therefore into the solution (32) to enter a chiral parameter it is irrational.

Obviously for the nonlinear equations (25) or (26) there should be a multiwave solution. Multiwave solutions are found for very much limited circle of the nonlinear wave equations $[16,17]$. The multiwave solution should depend on concentration of the chiral elements in a plate. Only with its help it is possible to understand under what conditions it is possible is proved to enter the chiral parameter, i.e. to understand borders of the material equations (1) - (4) applicability.

The equation (25) supposes the multiwave solution as:

$$
\varphi=\varphi_{0}+\varphi_{\max } \sum_{n=1}^{N} \exp \left(-\frac{\left(k_{0}\left(X-X_{0 n}\right)-\omega_{0}\left(t-t_{0 n}\right)\right)^{2}}{2}\right),
$$

where $N$ there are quantity of the waves-impulses kept within a length $l$ of a plate, fig. 2 , equal to number of the chiral elements, $n$ - current number of an impulse, $X_{0 n}$ - coordinates of waves-impulses maxima, $t_{0 n}$ - times of these maxima achievement.

Substituting (33) in (25) we shall find:

$$
V^{2}\left(\frac{\partial^{2} \varphi}{\partial X^{2}}\right) \sum_{n=1}^{N} \varphi_{n}+\omega_{0}^{2}\left(\sum_{n=1}^{N} \varphi_{n}\right)^{2}=\left(\frac{\partial \varphi}{\partial t}\right)^{2} .
$$

where it is designated:

$$
\varphi_{n}=\exp \left(-\frac{\left(k_{0}\left(X-X_{0 n}\right)-\omega_{0}\left(t-t_{0 n}\right)\right)^{2}}{2}\right) .
$$

Finding the derivatives on coordinate $X$ : 


$$
\begin{aligned}
& \frac{\partial^{2} \varphi}{\partial X^{2}}=\sum_{n=1}^{N}\left(\varphi_{n}\left(k_{0}\left(X-X_{0 n}\right)-\omega_{0}\left(t-t_{0 n}\right)\right)^{2} k_{0}^{2}+\varphi_{n} k_{0}^{2}\right)= \\
& =k_{0}^{2} \sum_{n=1}^{N} \varphi_{n}\left(k_{0}\left(X-X_{0 n}\right)-\omega_{0}\left(t-t_{0 n}\right)\right)^{2}+k_{0}^{2} \sum_{n=1}^{N} \varphi_{n}
\end{aligned}
$$

and on time $t$ :

$$
\frac{\partial \varphi}{\partial t}=-\omega_{0} \sum_{n=1}^{N} \varphi_{n}\left(k_{0}\left(X-X_{0 n}\right)-\omega_{0}\left(t-t_{0 n}\right)\right),
$$

we substitute (36) and (37) in the equation (34) and taking into account $k_{0} V=\omega_{0}$ we have:

$$
\begin{aligned}
& \left(\sum_{n=1}^{N} \varphi_{n}\left(k_{0}\left(X-X_{0 n}\right)-\omega_{0}\left(t-t_{0 n}\right)\right)^{2}+\sum_{n=1}^{N} \varphi_{n}\right) \sum_{n=1}^{N} \varphi_{n}= \\
& =\left(\sum_{n=1}^{N} \varphi_{n}\right)^{2}+\left(\sum_{n=1}^{N} \varphi_{n}\left(k_{0}\left(X-X_{0 n}\right)-\omega_{0}\left(t-t_{0 n}\right)\right)\right)^{2} .
\end{aligned}
$$

Reducing in the left and right parts (38) the identical addends $\left(\sum_{n=1}^{N} \varphi_{n}\right)^{2}$ we shall find:

$$
\begin{aligned}
& \sum_{n=1}^{N} \varphi_{n} \sum_{n=1}^{N} \varphi_{n}\left(k_{0}\left(X-X_{0 n}\right)-\omega_{0}\left(t-t_{0 n}\right)\right)^{2}= \\
& =\left(\sum_{n=1}^{N} \varphi_{n}\left(k_{0}\left(X-X_{0 n}\right)-\omega_{0}\left(t-t_{0 n}\right)\right)\right)^{2}
\end{aligned}
$$

Let's consider two one after the other going the identical impulses $n=1,2$. Writing down for this case the formula (39) we shall find:

$$
\begin{aligned}
& \left(\varphi_{1}+\varphi_{2}\right)\left(\varphi_{1}\left(k_{0}\left(X-X_{01}\right)-\omega_{0}\left(t-t_{01}\right)\right)^{2}+\varphi_{2}\left(k_{0}\left(X-X_{02}\right)-\omega_{0}\left(t-t_{02}\right)\right)^{2}\right)= \\
& =\left(\varphi_{1}\left(k_{0}\left(X-X_{01}\right)-\omega_{0}\left(t-t_{01}\right)\right)+\varphi_{2}\left(k_{0}\left(X-X_{02}\right)-\omega_{0}\left(t-t_{02}\right)\right)^{2}\right.
\end{aligned}
$$

Transforming the formula (40) we shall receive:

$$
k_{0}\left(X_{02}-X_{01}\right)-\omega_{0}\left(t_{02}-t_{01}\right)=0 .
$$

The formula (41) shows that distance between chiral elements $\delta=\left(X_{02}-X_{01}\right)$, fig. 2, an electromagnetic impulse propagates in time $\left(t_{02}-t_{01}\right)$ with a speed $V=\frac{\omega_{0}}{k_{0}}$. The size $\frac{1}{\delta}$ characterizes linear concentration of the chiral elements in a plate.

Using in (39) $\quad t_{0 n}=\frac{X_{0 n}}{V}=\frac{k_{0} X_{0 n}}{\omega_{0}}$, we receive that expressions in brackets $\left(k_{0}\left(X-X_{0 n}\right)-\omega_{0}\left(t-t_{0 n}\right)\right)^{2}=\left(k_{0}(X)-\left(\omega_{0} t\right)\right)^{2}$ do not depend from $n$ they can be taken out for a symbol of the sum and to reduce. In result (39) turns to identity.

Hence (33) is the multiwave solution of the nonlinear equation (25).

The most simple kind the multiwave solution (33) has in occasion of identical distance between all impulses and accordingly between of the chiral elements. In this case coordinates of maxima of impulses are $X_{0 n}=n \delta$, and times of achievement of maxima $t_{0 n}=\frac{k_{0} X_{0 n}}{\omega_{0}}=\frac{k_{0} n \delta}{\omega_{0}}$.

On fig. 3 for an illustration the some impulses following one after another plotted under the formula (33) are shown under conditions of the dimensionless sizes: $V=0$ - absence of dependence on time (the figure fixed in time) $\varphi_{0}=0, \varphi_{\max }=1, k_{0}=2, \delta=4$. 


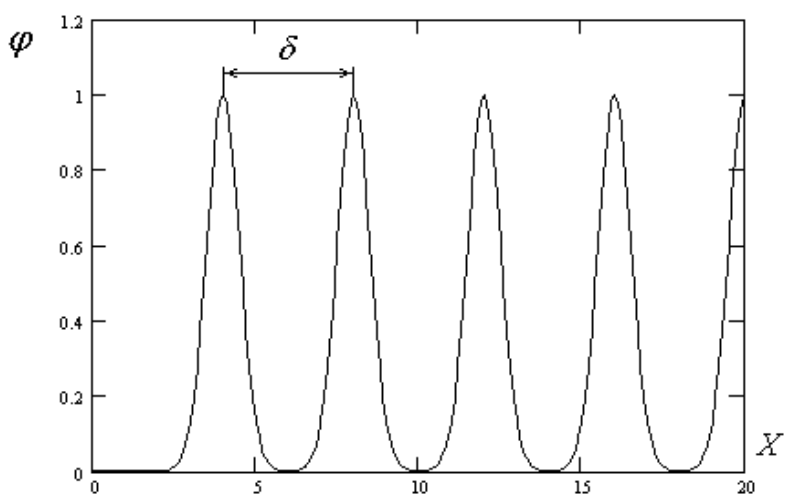

Figure 3. The impulses following one after another in the multiwave solution

Thus the formula (33) under condition of uniform distribution of identical impulses is the multiwave periodic solution of the nonlinear equation (25).

\subsection{The Solution as Standing Waves}

Let's consider in more detail another kind of the wave arising on single-row chiral plate at falling on it of an electromagnetic wave.

Standing waves are formed in linear systems as a result of superposition (interference) of the direct and reflected traveling waves more often. However it is known that standing waves can arise in nonlinear systems [18]. Many physical processes have essentially nonlinear character and process of standing waves occurrence in such systems is nontrivial. We shall examine an opportunity of standing waves occurrence in researched chiral medium.

The nonlinear equations (25) and (26) can be solved by a method of the Fourier variables division [19]. We search the solution of the equation (25) as:

$$
\varphi-\varphi_{0}=\varphi(X) T(t)
$$

where $\varphi(X)$ there is function only coordinates $X, T(t)$ - a function only time $t$.

Having substituted (42) in (25) we shall find:

$$
V^{2} \varphi(X) T^{2}(t) \frac{\partial^{2} \varphi(X)}{\partial X^{2}}=\left(\varphi(X) \frac{\partial T(t)}{\partial t}\right)^{2}-\varphi^{2}(X) T^{2}(t) \omega_{0}^{2} .
$$

Let's divide both parts of the equation on $\varphi^{2}(X) T^{2}(t)$. In result we shall receive:

$$
V^{2} \frac{1}{\varphi(X)} \frac{\partial^{2} \varphi(X)}{\partial X^{2}}+\omega_{0}^{2}=\left(\frac{1}{T(t)} \frac{\partial T(t)}{\partial t}\right)^{2}=-\alpha^{2}
$$

where $\alpha$ there is a constant.

The equation (44) breaks up to two independent equations. The equation dependent on $X$ looks like:

$$
\frac{\partial^{2} \varphi(X)}{\partial X^{2}}+\left(k_{0}^{2}+\frac{\alpha^{2}}{V^{2}}\right) \varphi(X)=0 \text {. }
$$

Comparing (45) and (27) we notice that $k_{S}^{2}=k_{0}^{2}+\frac{\alpha^{2}}{V^{2}}$. Hence $k^{2}=\frac{\alpha^{2}}{V^{2}}$, and hence $\alpha=\omega$.

The solution of the equation (45) we shall write down as:

$$
\varphi(X)=\varphi(0) \exp \left(i k_{S} X\right)
$$

where $\varphi(0)$ there is value of function $\varphi(X)$ in the beginning of coordinates.

The second equation of equality (44) looks like: 


$$
\frac{\partial T(t)}{\partial t}=i \omega T(t)
$$

Solving this equation we shall find:

$$
T(t)=T(0) \exp (i \omega t)
$$

where $T(0)$ there is initial value of function $T(t)$.

Using (42), (46) and (48) we shall find the solution of the equation (25):

$$
\varphi-\varphi_{0}=\varphi_{A} \exp (i \omega t) \exp \left(i k_{S} X\right)
$$

where it is designated $\varphi_{A}=T(0) \varphi(0)$ - a peak value of potential $\varphi-\varphi_{0}$ on a plate.

The function $\varphi-\varphi_{0}$ should not have imaginary addends, the potential is real size. Use an exponents with imaginary parameters is entered for convenience of transformations. Really in these exponents it is necessary to take into account only real items. Therefore the formula (49) describes the solution of the equation (25) as standing waves:

$$
\varphi-\varphi_{0}=\varphi_{A} \cos \omega t \cos k_{S} X=\varphi_{A} \cos \omega t \cos \frac{2 \pi X}{\delta},
$$

where $\varphi_{A}$ there is a peak value of standing waves, $\delta$ - length of a wave.

Condition of the nodes occurrence in a standing wave $X_{\mathrm{ns}}= \pm(2 n+1) \frac{\delta}{4}$, where $n=0,1,2, \ldots$.

On the ends of the single-row chiral plate, fig. 2 , should be nodes of a standing wave. If excitation of a wave occurs in the center of a plate the number of the maximal distant node from a center of a plate can be found under the formula $\pm \frac{l}{2}= \pm\left(2 n_{\max }+1\right) \frac{\delta}{4}$ or $n_{\max }=\left(\frac{l}{\delta}-\frac{1}{2}\right)$.

It is necessary to note that running waves $\varphi-\varphi_{0}=\frac{\varphi_{A}}{2} \cos \left(k_{S} X \pm \omega t\right)$ with account $k_{S}^{2}=\frac{\omega_{0}^{2}+\omega^{2}}{V^{2}}$ are not the solution of the equation (25) therefore the formula (50) from the physical point of view cannot be presented as a sum of the direct, and reflected from borders plate waves though mathematical this procedure is simple for making. It is consequence of the equation (25) nonlinearity.

It is interesting to track graphically a transition of the multiwave solution (33) in the solution as standing waves (50). This transition is carried out at rapprochement of impulses, fig. 2, 3, i.e. at reduction of size $\delta$.

On fig. 4 the two curves are shown. Curve 1 is plotted under the formula (33) under conditions: $V=0, \varphi_{0}=0, \varphi_{\max }=1, k_{0}=2, \delta=2$ for $N=8$ impulses. Curve 2 is plotted (dotted line) under the formula (50) under conditions $\varphi_{0}=0,65$ and $\varphi_{A} \cos \omega t=0,38$ for some moment of time $t$.

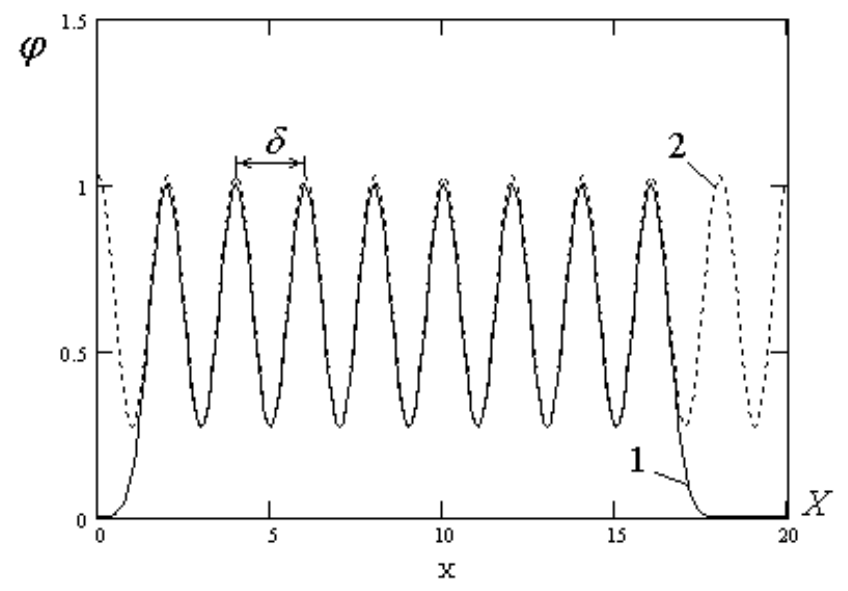

Figure 4. Transition of the multiwave solution in the solution as a standing wave: 1 - the multiwave solution, 2 - a standing wave 


\section{Conclusion}

Distribution of potential to a plate from a metamaterial with inductive chiral inclusions is investigated as with use of the material equations together with the Maxwell's equations, and on the basis of a detailed method of calculation of the chiral elements and an electromagnetic wave interaction. Comparison of two approaches has allowed to find out that introduction of the chiral parameter is correct only at enough high concentration of the chiral inclusions. On the basis of comparison of two methods results the frequency dependence of chiral parameter is found. At use of a detailed method of calculation the nonlinear equation for the potential having solutions as standing waves and solitary waves is received. Traveling waves are not the solution of this equation. At reduction of distance between chiral elements the process of transition of the multiwave solution of the nonlinear equation in the solution as a standing wave is investigated.

\section{References}

1. Slusar V. Metamaterials in the antennas techniques: a history and main principles. Minsk, Electronics, NTB. 2009, No. 7, P. 70-79.

2. Capolino F. Theory and Phenomena of Metamaterials. Boca Raton, Taylor \& Francis, 2009. 992 p.

3. Vendik I.B., Vendik O.G. Metamaterials and its Application in Technique of Ultrahigh Frequencies. SPetersburg. Tech. Phys. 2013, V. 58, No. 1, P. 1-24.

4. Davidovich M.V. Hyperbolic Metamaterials: Production, Properties, Applications, and Prospects. Uspekhi Fizicheskikh Nauk, 2019, V. 189, No. 12, Pp. 1249-1284.

5. Neganov V.A., Osipov O.V. Reflecting, Waveguiding, and Emitting Structures with Chiral Elements. Moscow, Radio i Svyaz, 2006, 280 p.

6. Katsenelenbaum B.Z., Korshunova E.N., Sivov A.N., Shatrov A.D. Chiral Electrodynamics Objects. Moscow, Uspekhi Fizicheskikh Nauk, 1997, V. 167, No. 11, Pp. 1201-1212.

7. Volobuev A.N. Electrodynamics of Circular Dichroism and its Application in the Construction of a Circular Polaroid. S-Petersburg. Tech. Phys. 2016, V. 61, No. 3, P. 337-341.

8. Levich V.G. Course of Theoretical Physics. V. 1. Moscow, Fizmatlit, 1962, 696 p.

9. Volobuev A.N. The Nonlinear Analysis of Chiral Medium. Ed. Takashiro Akitsu "Chirality from Molecular Electronic Ststes". IntechOpen. 2018. P. 1-10.

10. Volobuev A.N. Spreading of Pulse of Electromagnetic Field in Dielectric for Conditions Self-Induced Transparency. Mathematical Models and Computer Simulations, 2006, V. 18, No. 3, P. 93-102.

11. Volobuyev A.N., Zhukov B.N., Ovchinnikov Ye. L., Bakhito A.U., Trufanov L.A. Non-Linear Modelling of the Spread of an Action Potential. 1991. Pergamon Press. Biophysics. Vol. 36, No. 3, pp. 545-550.

12. Volobuev A.N. Inductance-Capacitor Model of an Excitable Biotissue. Uspekhi sovremennoi radioelectroniki. 2006. No. 3, pp. 33-60.

13. Condon E. Theory of optical rotating ability. Moscow, Uspekhi Fizicheskikh Nauk, 1938, V. 19, No. 3, Pp. 380430.

14. Volobuev A.N. Quantum Electrodynamics through the Eyes of a Biophysics. New York, Nova Science Publishers, Inc., 2017. 252 p.

15. Volkenchtein M.V. Biophysics. S-Petersburg, Lan, 2008, 596 p.

16. Ablowitz M.J., Segur H. Solitons, and the Inverse Scattering Transform. Philadelphia: SIAM, 1981.

17. Dodd R.K., Eilbeck J.C., Gibbon J.D., Morris H.C. Solitons and Nonlinear Wave Equation. London, New York, Tokyo: Academic Press, Inc., 1984.

18. Krasilnikov V.A., Krylov V.V. Introduction in physical acoustics. M.: Nauka, 1984. 403 p.

19. Tikhonov A.N., Samarski A.A. Equipments of Mathematical Physics. Moscow, Nauka, 1972. 736 p. 